\title{
Declining Use of Orthotopic Reconstruction for Bladder Cancer
}

\author{
Richard Hautmann, MD and Christian Bolenz, MD \\ University of Ulm, Ulm, Germany
}

Groeben et al. should be congratulated for tackling challenging and delicate issues: $:^{1}$ first, the huge discrepancy in use of continent diversion (CD) between Germany and the USA, and second, the declining use of orthotopic reconstruction. However, some important points must be made:

1. The authors' statement that the share of patients with incontinent urinary diversion (UD) remained stable in the USA does not seem to be valid. In a very recent publication, Farber et al. report declining use of $\mathrm{CD}$ since 2008. ${ }^{2}$ They studied contemporary trends in utilization of ileum conduit (IC) and CD in patients undergoing radical cystectomy (RC). Also using the National Inpatient Sample 2001-2012, they identified all patients diagnosed with bladder cancer (BC) who underwent $\mathrm{RC}$ followed by IC or $\mathrm{CD}$, revealing that 69,049 ICs and 6991 CDs were performed. CDs increased from 2001 to 2008 but declined after 2008 $(p<0.0001)$. Patients of all ages received ICs at a higher rate than CDs.

2. The authors correctly describe major differences in the way medicine and urology are practiced and reimbursed in the USA versus Germany. In Germany, $>80 \%$ of patients with muscle-invasive BC (MIBC) receive $\mathrm{RC}$, whereas half of US patients do not receive any curative treatment. Also, $30 \%$ receive radiation or chemotherapy, and only about $20 \%$ of patients with T2 MIBC undergo RC. This is a major source of bias in the analysis. ${ }^{1}$

(C) Society of Surgical Oncology 2018

First Received: 27 February 2018;

Published Online: 18 July 2018

R. Hautmann, MD

e-mail: richard.hautmann@uni-ulm.de
3. In Germany, the majority of BC patients are operated at high-volume institutions. The patients are diagnosed by urologists in private praxis. They refer the patient to an institution of their choice with a treatment plan, which is usually adhered to by the hospital. This leads to the $40 \% \mathrm{CD}$ rate in Germany and nations with similar systems, e.g., Austria. This situation is the reason why the rates of continent UD are four times higher in Germany than in the USA, where continent UD is widely underused (7.5 vs. $33.2 \%$ ).

4. Both the Groeben article ${ }^{1}$ as well as the Faber article ${ }^{2}$ try to analyze the declining use of continent UD. Limited by the nature of population-based datasets, but nevertheless unanimously, they report age, sex, comorbidity, use of robotics, reimbursement, and longer surgical time as causative. Moreover, in the USA, only $30 \%$ of all graduating urology residents performed more than 20 UDs of any type. As a consequence, many surgeons may lack comfort in not only performing $\mathrm{CD}$ but also postoperative management. Teaching hospitals, especially National Cancer Institute (NCI)-designated cancer centers, might also have nursing and support staff that are more familiar with CDs, allowing surgeons to feel more comfortable performing them.

5. Recently, this situation was addressed by an editorial comment in The Journal of Urology: "Worldwide declining use of orthotopic reconstruction-what went wrong?" 3 The three key drivers for the decreasing orthotopic reconstruction rate are (1) 50\% of all radical cystectomies for bladder cancer are done by low-volume surgeons, (2) imperfect surgical technique leads to imperfect functional results, and (3) in robotic-assisted radical cystectomy, early in the learning curve, achieving oncologic efficacy was the main priority. As a consequence, the orthotopic reconstruction rate following robotic surgery is at best $30 \%$ of that of open surgery. 
Taken together, prospective studies are required to further elucidate the many factors resulting in disparate use of UD.

\section{REFERENCES}

1. Groeben C, Koch R, Baunacke M, Schmid M, Borkowetz A, Wirth MP, et al. Urinary diversion after radical cystectomy for bladder cancer: comparing trends in the US and Germany from 2006 to
2014. Ann Surg Oncol. 2018. https://doi.org/10.1245/s10434-0186381-1.

2. Faber NJ, Faienna I, Dombrovskiy V, Tabakin AL, Shinder B, Patel R, et al. Disparities in the use of continent urinary diversion after radical cystectomy for bladder cancer. Bladder Cancer. 2018;4:113-120.

3. Hautmann R. Worldwide declining use of orthotopic reconstruction-what went wrong? J Urol. 2018;199:900-903. 